\title{
LA EXPRESIÓN CORPORAL Y LA TRANSVERSALIDAD COMO UN EJE METODOLÓGICO CONSTRUIDO A PARTIR DE LA EXPRESIÓN ARTÍSTICA
}

\author{
Consuelo Arguedas Quesada
}

\begin{abstract}
Recibido 26-V-2003 • Aceptado 12-VIII-2003
"Las formas expresivas han tenido desde siempre, la habilidad de transformar el medio, proporcionando una lectura simbólica y creativa que permite crecer; el sentido lúdico que subyace en ellas es el mejor substrato para la modificación cultural”.
\end{abstract}

(Porta, 1999, p.650)

\begin{abstract}
Resumen: La expresión corporal es una disciplina que le ofrece al ser humano la posibilidad de comunicar sentimientos, estados de ánimo, emociones, conocimientos y sensaciones de manera creativa y que hacen referencia a la parte subjetiva del hombre y de la mujer. Le permite manifestarse de manera espontánea a partir del conocimiento del cuerpo en forma individual o grupal, teniendo en cuenta las impresiones que perciba a través de los sentidos y su relación con el espacio y el tiempo, además le permite fortalecer la autoestima del individuo.

Para obtener prácticas adecuadas de expresión corporal, es necesario tener en cuenta el manejo apropiado de los estímulos o consignas, así como de los materiales utilizados, los recursos y la diversidad de actividades creativas.

La expresión corporal favorece el desarrollo armónico del ser mediante estímulos biológicos, intelectuales y socioemocionales, que le facilitan el proceso creativo y de sensibilización, admitiendo además, que la persona actúe en relación consigo misma, con otras personas, con otros seres vivos y con los objetos.

$\mathrm{Al}$ ofrecer beneficios para el desarrollo integral del hombre, la expresión corporal se convierte en un eje transversal en la educación primaria, al consentir la integración de contenidos de las distintas áreas del currículum escolar. Mediante estrategias novedosas se establece una interrelación entre los objetivos y se transforman los contenidos temáticos en aprendizajes significativos, que benefician la creatividad y por ende la expresión artística de los estudiantes.
\end{abstract}

Palabras clave: Expresión Corporal, Transversalidad, Expresión Artística, Educación Primaria.

\section{Introducción}

El propósito de este artículo es destacar el valor didáctico de la expresión corporal para ser integrada en el currículo escolar. El tema se deriva de la investigación bibliográfica de la autora y del proyecto de investigación ya concluido, "La expresión artística: un recurso heurístico para la construcción de procesos pedagógicos desde una perspectiva de género" número 724-A1-083, que se realizó en una escuela de atención prioritaria.

En él se define el concepto de la expresión corporal, se mencionan los objetivos generales y sus características específicas; también se hace referencia a los temas transversales como recurso metodológico, que permite la aplicación de la expresión corporal en la escuela.

Hoy día no se concibe que esta forma de expresión requiera aptitudes especiales, ni que sea privilegio de unos pocos; por el contrario, el ser humano necesita de ella sin excepción. Es el medio idóneo para promover el desarrollo intelectual, físico, sensorial y emocional, utilizando técnicas que favorecen a la vez la atención, la concentración y 
el elemento creativo; aspectos que son necesarios también en las diversas áreas del conocimiento. Estas experiencias son aceptadas por las niñas y los niños con gran beneplácito porque les ayudan a descubrir su subjetividad y el disfrute de la recreación.

\section{Expresión corporal}

La expresión corporal busca facilitarle al ser humano el proceso creativo y de libre expresión y comunicación, a partir del conocimiento de su cuerpo, del manejo del espacio, de los materiales y del fortalecimiento de su autoconfianza. Para el logro de una expresión placentera, es importante la motivación que el estudiante reciba de los docentes o adultos y del trabajo con sus compañeros y compañeras.

El objetivo primordial de la expresión corporal con niños y niñas surge del respeto a la unicidad de cada persona y de las diversas maneras de aprender; por esta razón se desarrolla la creatividad y el autoconocimiento, con el fin de fortalecer la autoestima, la sensibilidad y la creación, teniendo en cuenta las limitaciones y las posibilidades de cada individuo.

En las clases de expresión corporal, la música sirve como elemento de apoyo, para que los infantes disfruten y muestren sus diferentes estados de ánimo; además les ayuda a conseguir una mejor comunicación. En estas sesiones, no puede faltar el placer del movimiento y el sentido lúdico, se liberan energías y se orientan hacia la expresión del ser. Por esta razón, su finalidad es contribuir a la integración de la persona, donde el cuerpo traduce el estado anímico del individuo, por medio de gestos, movimientos o quietud del cuerpo, con mensajes silenciosos o sonoros, individuales o colectivos. "El movimiento corporal se configura como un lenguaje propio sujeto a las condiciones fisiológicas, anímicas y espirituales que determinan el conjunto de su expresión”. (Riveiro, 1997, p. 74). Este lenguaje integra desde las funciones básicas para la supervivencia, hasta aquellas que por su significado o por su intención, benefician a las personas en el desarrollo integral.

La educación del movimiento traduce en el espacio, mediante la acción, los ritmos percibidos a través del oído, es decir, se coordinan los movimientos y las actitudes de los estudiantes con el estímulo sonoro. Por ello, un acompañamiento rítmico melódico como fondo de cualquier ejercicio, favorece la realización de este. El alumno recrea con el movimiento los ritmos, tempos o matices musicales que escucha, a la vez que desbloquea sus propias potencialidades expresivas, explorando las posibilidades de improvisación y de creación.

La percepción se lleva a cabo por medio de diferentes vías que generan categorizaciones intuitivas y sensoperceptuales, ya que en la expresión corporal se perciben sensaciones a través del tacto y de la piel al utilizar materiales con diferentes texturas, tamaños, colores, formas y la relación del objeto con el espacio y el tiempo.

De esta manera, al sentir el objeto se explora y se somete a diversas acciones, que favorecen la construcción del pensamiento de los estudiantes. La capacidad de representación se relaciona con lo observado y lo percibido por el individuo, dándose una etapa donde se repite, se imita, se lleva a cabo la improvisación de movimientos, hasta llegar a la creatividad con el juego simbólico.

La creación de estímulos y situaciones favorece la liberación interior y sirve para orientar la expresión con toda plenitud y espontaneidad, de acuerdo con el sentir del individuo. Las acciones no son impuestas, sino sugeridas por los estímulos o consignas, las cuales provocan la corporización de la imagen, conjuntamente con la expresión. Los estímulos pueden ser: ritmos ejecutados con instrumentos de percusión, frases, rimas, adivinanzas, poesías, canciones, 
juegos de sorteo, melodías, cuentos y otros. La asimilación de los estímulos y la forma en que reaccionan los estudiantes ante ellos, depende del uso adecuado de la voz, del instrumento musical empleado, del material que se utilice y de la motivación de las actividades.

\subsection{Beneficios de la expresión corporal}

Esta área favorece el crecimiento integral de los niños y de las niñas, de manera individual o colectiva en los aspectos socio-emocionales, intelectuales y biológicos. Stokoe (1976) considera que la expresión corporal proporciona los siguientes beneficios al ser humano:

\section{$1 \quad$ Desarrollo de la capacidad} de actuar

El movimiento es una cualidad primordial que para el niño y la niña escolar, ofrece infinitas posibilidades en su incursión del mundo que los rodea, mediante la experimentación real, concreta y directa con su propio cuerpo.

\section{$1 \quad$ Estímulos individuales y colectivos}

El niño y la niña elaboran y expresan ideas y sentimientos según sus propias características, lo cual ayuda al desarrollo individual y personal, y al mismo tiempo favorece la conciencia grupal.

\section{$1 \quad$ Estímulos biológicos}

La expresión corporal responde al desarrollo armónico del cuerpo en relación con la salud, al mismo tiempo con la postura, la respiración, la coordinación, el reposo y la motricidad, que favorecerá el aprendizaje de la escritura.
$1 \quad$ Estímulos intelectuales

Se refuerza el conocimiento del esquema corporal y la respectiva diferenciación del cuerpo con los objetos que lo rodean, la adquisición de las constantes espaciales y temporales, elementos de cantidad, la toma de conciencia con la realidad, la noción de los objetos y su posición en el espacio.

\section{$1 \quad$ Desarrollo socioemocional}

Se estimula el trabajo en grupos unificados por la expresión de una idea común, para lo cual son de gran importancia las rondas y juegos con compañeros y compañeras, donde se emplea un lenguaje diferente como medio de comunicación y el aprecio a los valores estético-musicales. De esta forma se ayuda a consolidar la autoestima, a partir del conocimiento del propio cuerpo y el de los demás, para aprender a percibirlo, quererlo y respetarlo.

$1 \quad$ Desarrollo de la sensibilidad y de la creatividad

Estos aspectos se benefician por medio de la variedad de estímulos sonoros y de la audición de obras de diferentes estilos, autores y períodos musicales. Una gama amplia permite el desarrollo del sentido estético de la creación.

\subsection{Características de la expresión corporal}

Stokoe y Harf, citados por Bonilla (2001), consideran que la expresión corporal está conformada por la sensopercepción, la motricidad, el espacio y las cualidades del movimiento.

La sensopercepción se refiere al desarrollo de los sentidos, en forma 
exterioceptiva (brinda información exterior de nuestro cuerpo); y propioceptiva (sensaciones de motricidad, ubicación del cuerpo y peso).

La motricidad y el tono: la primera es definida por las autoras como "movimiento corporal". El tono lo describen como "el grado de tensión y de relajación muscular, así como también el equilibrio establecido entre ambos en momentos de acción y de reposo".

El espacio: puede ser personal, parcial, total y social. El primero es aquel que rodea nuestro cuerpo: el personal es el que ocupa nuestro cuerpo y los espacios interiores del mismo, el total facilita el desplazamiento y el social permite compartir con los demás.

Las características del movimiento: permiten clasificar las acciones en tres grupos: los movimientos naturales, los analíticos o técnicos y los generadores. Los naturales son aquellos que se realizan sin ninguna dificultad y en forma natural para que una persona física y mentalmente sana los pueda hacer; es decir, son los movimientos que se llevan a cabo en forma natural. Por ejemplo caminar, correr, saltar, deslizar, rodar y cuadrupedia.

Los movimientos técnicos son los que permiten localizarse en diferentes partes del cuerpo y se dividen en rotaciones, flexiones, estiramientos, contracciones, relajamientos y ondulaciones.

Los generadores se refieren a las cualidades de los movimientos, es decir, éstas pueden hacer cambiar el significado de los mismos, al tomarse en cuenta distintas variables como la lentitud, la rapidez, la suavidad, la fuerza, si los movimientos son directos o indirectos, fluidos o contenidos, por este motivo, están en relación con el tiempo al incursionar en el campo temporo-espacial.

Es importante recordar además que Stokoe y Harf (1992) consideran que la expresión corporal abarca cuatro niveles:

1. La persona en relación consigo misma.

2. La persona en relación con otras personas.

3. La persona en relación con los seres vivos.

4. La persona en relación con los objetos.

En el ser humano existe un paralelismo entre el desarrollo de las funciones motrices y las funciones psíquicas, el cual puede ser percibido cuando se desarrollan las habilidades motrices y se aumenta la exactitud y la economía de movimientos. Esto proporciona control personal, seguridad en sí mismo y con otras personas, con otros seres y con los objetos.

Las experiencias de expresión corporal con estudiantes de educación primaria favorecen la acción de alguna parte en especial del cuerpo o en su totalidad, pero también dan importancia a la relajación y al reposo. Es indispensable que se intercambien actividades de mucha acción con otras que inviten al descanso y a la calma.

Para una adecuada expresión del movimiento, es necesario que los ejercicios despierten la atención constante de los participantes, utilizando explicaciones claras, previas a la ejecución, pero sin dar el ejemplo; más bien dejándolo que se involucren la iniciativa y la creatividad; para que los copartícipes resuelvan la consigna solicitada al expresarse con libertad. La persona que dirige la actividad es sólo un motivador y un guía en el proceso, nunca debe convertirse en el ejemplo de la actividad que se va a realizar, pues limita el desarrollo creativo de los involucrados en el proceso. 
1.3. Objetivos generales de la expresión corporal

$1 \quad$ Contribuir en el desarrollo integral del ser humano en sus aspectos afectivos, cognitivos y psicomotrices.

$1 \quad$ Potenciar la capacidad expresiva de las personas para mejorar la comunicación del mundo interno y del externo.

$1 \quad$ Favorecer el desarrollo de individuos sensibles y creativos en forma individual y grupal respetando las diferencias de cada persona.

$1 \quad$ Lograr una mejor comunicación entre los seres humanos.

$1 \quad$ Estimular la creación de proyectos comunes, la necesidad de colaboración y el placer de la acción grupal.

1 Estimular la creatividad utilizando materiales auxiliares y de desecho con el fin de facilitar la auto-expresión.

\section{La expresión corporal como eje transversal en la educación primaria}

En el proceso escolar es necesario trabajar con la población estudiantil, de manera coordinada con los conocimientos de las diferentes disciplinas, unidos a juicios críticos y de valor, basados en las realidades y problemas de la vida diaria de las niñas y de los niños; es decir, se debe hacer referencia a los ejes transversales. Es aquí donde la expresión corporal juega un papel muy importante como área capaz de integrar y de enriquecer los contenidos del currículo escolar, mediante actividades lúdicas relacionadas con matemáticas, ciencias, estudios sociales, español, religión, artes plásticas, inglés u otras áreas del quehacer educativo. El uso adecuado de materiales auxiliares, instrumentos musicales y diversas prácticas donde se utilice el movimiento corporal, van a favorecer el logro de los objetivos propuestos de manera creativa y amena. Como consecuencia, la expresión corporal se convierte en un eje transversal que ofrece múltiples posibilidades para la educación primaria. Por este motivo es fundamental establecer la relación entre los objetivos y contenidos de las áreas específicas, con los objetivos y contenidos de cada uno de los temas del eje transversal. Esta unión permitirá enriquecer los contenidos y así convertirse en aprendizajes significativos.

"Las áreas quedarán enriquecidas y redimensionadas desde la realidad que los alumnos y las alumnas viven, consiguiendo así un mayor nivel de significatividad”. (González, 1999, p. 33).

Con el fin de ilustrar el planteamiento de este ensayo, se diseña una propuesta en la que los estudiantes abordan hechos de la vida cotidiana transformando los aprendizajes que han adquirido en el currículo escolar, en instrumentos útiles para su quehacer diario; ayudándole a la vez, en la adquisición de valores y actitudes. En esta transformación es donde la expresión corporal contribuye a que los contenidos temáticos sean reveladores, mediante estrategias novedosas y dinámicas, que favorecen el aprendizaje; es decir, actúa como eje transversal en el proceso educativo y establece coherencia e interrelación entre los objetivos del currículo escolar y los del eje transversal; en esta dinámica participativa se trabaja en forma globalizada e interdisciplinaria.

"Los temas transversales no se incorporan al currículo en forma forzada, sino todo lo contrario: son concreciones curriculares que surgen de la realidad cotidiana y de los problemas sociales y que, conectando con los objetivos generales del currículo escolar, los llenan de significado." (González, 1999, p. 59).

La expresión corporal se engloba dentro de la educación artística, donde el 
elemento expresivo y creativo, al igual que la comunicación de sentimientos, ideas y vivencias, complementan la elaboración de un trabajo final. Al aplicar los conocimientos artísticos a las experiencias más significativas de la realidad cotidiana, se logran actividades útiles y expresivas que contribuyen al goce y al bienestar personal. Se favorece la interiorización y la expresión de sentimientos, que unidos a la belleza, a la armonía y a la relajación sirven de base para desarrollar la criticidad de los estudiantes y contribuyen en su calidad de vida.

Esta área favorece también el respeto hacia los demás, con las posibilidades y limitaciones de cada individuo, poniendo en juego la cooperación y el trabajo en equipo, la aceptación del otro y la distribución igualitaria de tareas.

Con el uso de materiales auxiliares elaborados con elementos desechables, la expresión corporal contribuye a reutilizar implementos que formarán parte de una creación artística. De esta forma, la transversalidad también hace referencia a la educación ambiental permitiendo que los estudiantes valoren el objeto lúdico construido y a la vez colaboren con la naturaleza, al evitar el uso excesivo de recursos y la contaminación del medio ambiente.

Como consecuencia se puede afirmar que la expresión corporal se relaciona con los objetivos de la educación para la paz, con los de igualdad, los de educación vial, educación del consumidor, educación para la salud y educación ambiental, entre otros. Dicha afirmación corresponde a que estos temas son también reflexiones o problemas de la educación actual y constituyen por lo tanto, ejes transversales en el proceso educativo.

A partir de este planteamiento y tomando como referencia el eje temático "Los seres humanos somos parte integrante de la Naturaleza", del Programa de Estudios de Ciencias del Primer Ciclo de la Educación General Básica, se plantea la siguiente estrategia metodológica. El contenido curricular corresponde a los componentes del entorno, donde se persigue que éstos sean observados por los estudiantes, los comparen y los clasifiquen como seres vivos y seres no vivos, con el fin de protegerlos.

\section{Estrategia metodológica}

Después de escuchar una obra musical, se comenta sobre los posibles ecosistemas que podrían describirse con esa melodía: animales que habitan, plantas, clima, seres inertes, entre otros. La descripción se relaciona con las características musicales específicas de la obra, a saber: timbres sonoros, velocidad, carácter, intensidad y otras cualidades que permitan establecer semejanzas con objetos, lugares o seres vivos.

$1 \quad$ Posteriormente se invita a que se desplacen como algunos de estos animales y que imiten con su cuerpo las formas, los tamaños y la manera de comportarse.

1 Se invita a que los estudiantes descubran sonidos producidos por el cuerpo y que imiten aquellos que son emitidos por los seres que habitan ese ecosistema.

Algunos estudiantes utilizan materiales auxiliares como papeles o cintas plásticas que mueven para producir el sonido del viento, del mar o de algún elemento específico del ecosistema propuesto por los estudiantes.

En conjunto se crea un texto cuya letra esté relacionada con la temática tratada.

Los estudiantes representan con el cuerpo y objetos de la naturaleza (ramas, palos, piedras y otros), el texto que han creado. 
Los escolares acostados en el suelo, escuchan un cassette con efectos sonoros ambientales, con el fin de que reposen tranquilamente.

Para finalizar la actividad se comenta sobre las sensaciones percibidas en forma individual y grupal.

$1 \quad$ La evaluación se llevará a cabo por medio de la observación y la participación individual y colectiva en las actividades corporales y sonoras. Se realiza además, el comentario sobre los seres vivos e inertes, y las posibles soluciones para disminuir y evitar la contaminación ambiental.

Con esta estrategia metodológica se pretende visualizar la transversalidad de la expresión corporal en el área de Ciencias para el Primer Ciclo de la Educación General Básica, al enriquecer el contenido curricular de los componentes del entorno. A saber, la interacción en los ecosistemas, los peligros que amenazan el planeta, el desarrollo de una actitud de responsabilidad y de protección hacia el medio ambiente. Las actividades corporales evidencian situaciones de la vida real, llevadas a la práctica utilizando el elemento lúdico como recurso inherente al comportamiento escolar. De esta manera, la expresividad por medio del movimiento del cuerpo se convierte en eje transversal en el proceso educativo escolar, proporciona a los estudiantes aprendizajes novedosos, creativos e integrales que fomentan a la vez, el desarrollo de actitudes y valores.

Con el fin de aportar otras experiencias educativas que ejemplifican la transversalidad de la expresión corporal en la educación primaria, se plantean algunos ejemplos que enriquecen el proceso de enseñanza y aprendizaje:

1 Teniendo como estímulo una melodía lenta con intensidad suave, se invita a los estudiantes a representar la germinación de una semilla desde el momento en que se siembra, hasta convertirse en un árbol que da vida a muchos seres.

Se hace referencia a las partes de la planta y a las distintas clasificaciones. Por ejemplo, se mencionan consignas que sirven de estímulo para la interpretación corporal:

- ¿ ¿cómo represento con mi cuerpo una hierba, un arbusto o un árbol?

- ¿ ¿cómo personifico la raíz, el tallo, las hojas y el fruto de esa planta? De esta manera los estudiantes interiorizan el estímulo y lo exteriorizan de forma creativa.

1 Con este mismo estímulo musical pero adjuntando además el uso de telas grandes (manteles, sábanas u otras), se representa el tema de la comunicación con seres extraterrestres o el contenido de la fecundación.

$1 \quad$ Los estudiantes colocan en el suelo cintas plásticas e imitan figuras geométricas (rectángulos, triángulos, círculos). Se desplazan realizando movimientos distintos (naturales generadores o analíticos), teniendo en cuenta además, la figura representada y el timbre sonoro escuchado. Así por ejemplo, al oír el sonido del pandero se mueven los alumnos que hubiesen representado con las cintas círculos, al escuchar el timbre del triángulo lo hacen los que imitaron la figura del triángulo y al escuchar el sonido de la caja china les corresponde a quienes representaron el rectángulo. En este caso, el objetivo pretende identificar figuras planas en cuerpos geométricos y clasificarlas por sus características de forma.

Cantar una canción relacionada con el tema de prevención de accidentes 
en la escuela o en el hogar y representarla corporalmente, o aplicar esta actividad al contenido de desastres naturales.

Realizar ejercicios de sensibilización donde los alumnos se colocan en parejas, con los ojos cerrados y con los manos abiertas frente a frente, para transmitir sensaciones mediante el tacto y de esta manera reforzar el contenido curricular de los sentidos; como parte del eje temático conozco mi cuerpo.

Interpretar con mimo un relato creado por los estudiantes donde se reflejen distintos valores y disvalores.

1 Representar en forma corporal los deberes y los derechos de las niñas y de los niños. Haciendo uso de pañuelos o cintas de tela para reforzar la creatividad individual y grupal

De esta manera, se evidencia que en todos los ejemplos mencionados se aplican contenidos de expresión corporal relacionados con improvisación de movimientos, imitación eco, imitación simultánea, creación, niveles, espacios y clases de movimientos. Al mismo tiempo que se hace referencia a objetivos y contenidos de $\mathrm{Ma}$ temática, Ciencias, Estudios Sociales y Español de los Programas de Estudios del Ministerio de Educación Pública.

\section{A manera de conclusión}

En la Educación Primaria la expresión corporal es indispensable para el desarrollo integral del contenido curricular. Con la integración de la música y la expresión corporal se motiva a los estudiantes y se redescubre la capacidad creativa de los alumnos y del docente; de esta forma se concibe al niño y a la niña como seres completos, con sus intereses y experiencias. El uso de rimas, adivinanzas, pregones, canciones, jitenjáforas (series de palabras sin un sentido o significado definido), juegos, rondas, danzas, expresiones folklóricas y todas las posibilidades que el entorno sonoro y el movimiento pueden ofrecer a una educación amena, interesante y divertida, facilita y desarrolla la creatividad y transforma la enseñanza en aprendizajes significativos.

Nuestra misión como educadores y educadoras consiste en brindar experiencias de expresión corporal de calidad, que abarquen el aspecto cognitivo, sensorial y afectivo de los niños y de las niñas y que permitan la integración con los otros contenidos escolares.

Partiendo de la aplicación que la autora realiza en su práctica pedagógica sobre esta temática y de la puesta en práctica en la escuela de atención prioritaria donde se llevó a cabo este proyecto de investigación, se concluye que la expresión corporal logra integrar los contenidos curriculares escolares y evita una educación memorística e indiferente, al emplearse como eje transversal en el proceso educativo. Los temas más diversos resultan ser de gran interés para los estudiantes, al proporcionárselos el docente en forma lúdica y no tradicional. Por ejemplo se pueden mencionar algunos de ellos que fueron puestos en práctica con los estudiantes y de manera satisfactoria, por las docentes del centro educativo donde se realizó la experiencia pedagógica: los mitos y las leyendas, las aves, la energía, el reloj, la educación sexual, los números pares e impares, las fracciones, la divisibilidad, los reinos biológicos, los ecosistemas y la narración de cuentos entre muchas otras posibilidades.

Teniendo en cuenta estas reflexiones, concluyo mi planteamiento afirmando con certeza, que la expresión corporal enriquece la labor pedagógica del docente y el desarrollo integral de la población estudiantil. Por estas razones, dejo abierta la invitación a todos los educadores para que se involucren en esta vivencia, ya que les 
dejará muchos momentos satisfactorios en el quehacer educativo y personal.

\section{Referencias bibliográficas}

Beuchat R. Cecilia, Desarrollo de la Expresión Integrada: Club Cli-Clo-Pips. Chile: Editorial Andrés Bello, 1993.

Bonilla Astorga, Yinia et al. La Expresión Corporal y la Música en el Currículo de Educación Inicial. Tesis de Grado. Ciudad Universitaria Rodrigo Facio. San José, 2001.

Eufonía 8. Creatividad-Improvisación. Didáctica de la Música. España: Editorial GRAÓ, 1997.

Eufonía 7, Procedimientos en la Educación Musical. Didáctica de la Música. Barcelona: Editorial GRAÓ, 1997.

Eufonía 14, La Música en la Educación Infantil. Didáctica de la Música. Barcelona: Editorial GRAÓ, 1999.

Frega, Ana Lucía. Música para Maestros. España: Editorial GRAÓ, 1998.
González Lucini, Fernando. Temas Transversales y Educación en Valores. Madrid: Grupo Anaya, 1998.

González Lucini, Fernando. Temas Transversales y Áreas Curriculares. Madrid: Tercera Edición. Grupo Anaya, 1999.

Pascual Mejía, Pilar. Didáctica de la Música. Madrid: Pearson Educación, 2002.

Programas de Estudios de Ciencias, Matemática, Español, Estudios Sociales. Primer Ciclo de Educación General Básica. San José: Ministerio de Educación Pública, 2001.

Sefchovich, Galia-Waisburd Gilda. Expresión Corporal y Creatividad. México: Editorial Trillas, 1997.

Vargas D., Ana Isabel. Técnicas para el Desarrollo de la Expresión y el Movimiento en la Escuela Primaria o Básica. Modulo 7 , Unidad 7,2 - Unidad 7,3. San José: Oficina Subregional de Educación de la UNESCO para Centroamérica y Panamá.

Consuelo Arguedas Quesada Investigadora del Instituto de Investigaciones para el mejoramiento la Educación Costarricense (IIMEC) 